\title{
ARMES IBÉRIQUES ENTRE ALMEDINILLA ET OXFORD, EN PASSANT PAR PARIS: LES DESSINS DE JEAN-CHARLES GESLIN EN 1870
}

\author{
POR \\ FERNANDO QUESADA y PIERRE ROUILLARD
}

\begin{abstract}
RÉSUMÉ - RESUMEN
Des armes ibériques en fer en bon état de conservation et provenant de fouilles anciennes sont actuellement conservées au Musée des Antiquités Nationales de Saint-Germain-en-Laye et au British Museum. A ces deux ensembles il convient d'ajouter le lot d'armes ibériques conservé aujourd'hui au Pitt Rivers Museum d'Oxford, que nous présentons ici et qui témoigne de l'intérêt pour l'Espagne antique de quelques antiquaires et collectionneurs français et anglais de la fin du XIXe siècle. Cet intérêt n'a pas seulement abouti à constituer des vitrines à Paris, Londres ou Oxford, mais a contribué à diffuser le goût pour la culture ibérique, ce dont témoigne, dans le domaine de l'armement, par exemple, la publication en 1913 de l'ouvrage de H. Sandars, The weapons of the Iberians.
\end{abstract}

Es un hecho que buen número de armas ibéricas de hierro en excelente estado de conservación y procedentes de excavaciones antiguas se encuentran hoy depositadas en museos extranjeros, como el de Saint-Germain-enLaye o el British Museum. A estos conjuntos puede añadirse el lote de armas ibéricas que presentamos, conservado hoy en el Pitt Rivers Museum de Oxford. Estas piezas tienen una historia interesante en sí misma; son exponentes del interés por la antigua Iberia de algunos investigadores y coleccionistas de final del s. XIX, fundamentalmente franceses y británicos, interés que no sólo llenó interesantes vitrinas en Paris, Londres u Oxford, sino que difundió el atractivo de la Cultura Ibérica y trajo consigo la edición de algunas de las obras más importantes de principios de siglo sobre su arqueología, como, en el caso de las armas, la del británico H. Sandars The weapons of the Iberians.

\section{MOTS CLÉS - PALABRAS CLAVE}

Culture ibérique. Arme. Collection archéologique. Historiographie.

Cultura Ibérica. Armas. Coleccionismo. Historiografía.

Dans la seconde moitié du XIXe siècle, de grandes collections archéologiques, celle de Campana, celle de Froehner, celles des frères Cesnola pour n'évoquer que les plus célèbres, se constituent, suscitées par ou suscitant de grandes explorations tant au Levant, qu'à Chypre ou en Etrurie, et alimentent la circulation d'objets. Les grands musées qui s'organisent dans la même période trouvent là un moyen d'enrichir leurs collections. Dans ce mouvement, de la fouille à la dispersion d'objets, l'Espagne est présente, mais bien modestement. Un témoignage de ces phénomènes est fourni par un ensemble de cinq planches de dessins au crayon d'armes ibériques effectués par Jean-Charles Geslin en 1870 et conservés dans les Bibliothè- 
ques et Archives des Musées Nationaux (Louvre) ${ }^{1}$. Le cas est exemplaire car l'essentiel de la chaîne, du lieu de provenance (Almedinilla) au dépôt dans un musée (Oxford) a pu être reconstitué; le commanditaire est connu (Pitt Rivers) et notre bonne fortune tient à la générosité des experts chargés de la vente (Rollin et Feuardent) qui autorisèrent Geslin à faire ces relevés que nous publions aujourd'hui.

\section{DES DESSINS D’ARMES IBÉRIQUES AU LOUVRE.}

A la date où furent effectués les dessins, les objets que nous reconnaissons aujourd'hui comme ibères étaient peu nombreux et circulaient peu ${ }^{2}$, surtout en dehors de l'Espagne qui venait d'être dotée (en 1867) d'un Museo Arqueológico Nacional. La seconde moitié du XIXe siècle voit naître l'intérêt pour les vestiges antiques autres que ceux de Grèce, d'Italie ou d'Egypte et parmi eux les antiques d'Espagne, en dehors des monnaies, des antiques que souvent l'on ne sait comment qualifier, occupent une faible place. En 1870, des sculptures du Cerro de los Santos dans la province d'Albacete ont déjà probablement été transportées en dehors de Yecla et la nécropole de Almedinilla (dans la province de Cordova) est explorée par Luis de Maraver y Alfaro depuis trois ans; le marché parisien accueille précisément des armes de cette nécropole andalouse que nous connaissons grâce aux dessins de Geslin.

Les cinq planches de dessins présentées ici sont l'œuvre de Jean-Charles Geslin (18141885), peintre ${ }^{3}$ et collectionneur devenu en 1863 «Inspecteur des travaux des Antiques et de la Sculpture moderne» au Musée du Louvre; un de ses principaux centres d'intérêt dans le domaine archéologique est la sculpture chypriote ${ }^{4}$ mais ses goûts sont variés comme en attestent les dessins qu'il a réalisés.

Deux ensembles de dessins doivent être distingués; le premier est constitué d'environ 350 dessins et aquarelles qui étaient dans son bureau du Louvre au moment de son décès, archivés ensuite dans la Bibliothèque où nous avons retrouvé la pochette, avec les cinq planches d'armes ibériques, objet de cette note. Les dessins les plus nombreux concernent des pièces étrusques, grecques (collection Campana), orientales, chypriotes.

Le second ensemble nous est connu par le Catalogue de vente de sa collection au moment de sa succession le 7 février $1888^{5}$. L'inventaire compte des tableaux de Geslin (surtout des monuments et paysages d'Italie), des peintures de divers auteurs, des dessins et aquarelles de Geslin (d'Italie et nombreuses églises de Paris), des projets personnels d'ameublement et de décoration parfois empruntés à l'art antique, des gravures et lithographies et un ensemble

1 MS 272, 2, pochette 12. Nous tenons à remercier Annie Caubet, Conservateur général, Conservateur en chef du Département des Antiquités Orientales du musée du Louvre qui fit connaître à l'un d'entre-nous (P.R.) cet ensemble, le Conservateur de la bibliothèque Madame Isabelle Le Masne de Chermont et Madame Amélie Lefébure pour leur accueil. Les photographies sont dues au talent d'André Pelle, ingénieur au CNRS, Maison René Ginouvès, Archéologie et Ethnologie, Nanterre.

2 Avant la découverte de ces dessins de 1870, le premier objet ibère (en dehors des monnaies) connu pour avoir circulé avant d'être acquis par un musée est un mastos d'argent portant une inscription : A. HÉRON DE VILLEFOSSE, «Vase d'argent trouvé en Espagne et portant une inscription ibérique», CRAI, 1892, p. 403-408. Sur l'histoire des collections françaises : Pierre ROUILLARD et alii, Antiquités de l'Espagne, Paris, 1997, p. 9-16.

3 Il fut élève à l'Ecole des beaux-arts, exposa au Salon de 1841 à 1878 et fut médaillé en 1845 pour Vue du Forum romain, état actuel. En peinture les évocations des monuments antiques et leurs ruines sont ses sujets favoris ; mais il s'est aussi attaché à des sujets plus proches, en particulier des réalisations d'architecture contemporaine.

4 J.-Ch. GESLIN est l'auteur d'un travail sur l'art chypriote «Etudes sur l'art chypriote» publié en articles dans une revue à la vie courte Le Musée Archéologique, I et II, 1876 et 1877. On connaît de lui aussi «Lettre à M. Léon de Rosny sur quelques monuments trouvés en divers points de la grande région comprise entre le Tigre et l'Euphrate», dans $M e^{-}$ moires de l'Athénée Orientale, 1871, p. 31-42.

5 Oeuvres et collections de J.-Ch. Geslin, Hotel Drouot, 7, 8 et 9 février 1888, par le ministère de $\mathrm{M}^{\mathrm{e}}$ Tual, Commissaire-Priseur assisté de MM. Rollin et Feuardent, de M. B. Lasquin et de M. S. Mayer. 
de 900 planches de «dessins et aquarelles archéologiques» dont on ignore le ou les acquéreurs. Dans ces dessins et aquarelles aujourd'hui dispersés, on retrouve pêle-mêle les multiples centres d'intérêt de Geslin, les terres cuites, les vases des collections Argyropoulos, Campana, Castellani, les pièces ramenées par la Mission Renan en Phénicie, et on voit bien la place essentielle des objets de Grèce, d'Italie, de Chypre, d'Orient et la place infime (15 planches) des «Armes gauloises» (de la région de Dijon). Il est également fait mention «d'Armes espagnoles, Campana, musée Borbonico, Naples», qui doivent être des objets de l'époque moderne. Ne trouve t-on pas là un instantané des centres d'intérêt d'un érudit s'adonnant au monde antique?

Les dessins de Geslin, ceux du premier comme ceux du deuxième ensemble, concernaient tout à la fois des pièces conservées au Louvre (des pièces du «musée Campana») ou au British Museum et, ce qui est tout à fait précieux, des pièces de collections privées ou des pièces mises en vente sur le marché. Parmi celles-ci, bon nombre dont les «armes rapportées d'Espagne» ont été dessinées chez Rollin et Feuardent, «experts en médailles et antiquités» et actifs marchands d'antiquités ${ }^{6}$.

\section{DES ARMES IBÉRIQUES À OXFORD.}

La destinée d'une partie des objets mis en vente est connue, la collection du général A. H. L. Fox Pitt Rivers (aujourd'hui au Musée Pitt Rivers d'Oxford), celle la même d'autres objets, chypriotes, mis en vente par Rollin et Feuardent qui venaient des collections de Luigi et d'Alessandro Cesnola. Les grandes étapes et les acteurs sont connus et seules nous manquent les dates précises (autour de juillet 1870) d'arrivée à Paris et d'acquisition des armes ibériques.

Inventaire des pièces ibériques Geslin, Manuscrit Louvre, 272, 2, pochette 12 et leur localisation actuelle:

A.- Espagne, armes anciennes, MMrs Rollin et Feuardent, fer. Geslin, Juillet 1870. (Fig. 1).

L.: 56, 5; 1. A/B: 5,3 ; E/F: 6, 1; C/D: 3.

Aujourd'hui au Pitt Rivers Museum, inv. 1884.24.124.

B.- Espagne, armes anciennes, MMrs Rollin et Feuardent, fer. Geslin, Juillet 1870. (Fig. 2).

L.: 59, 5; 1. A/B: 3, 1; C/D: 5, 4.

Lieu de conservation actuel inconnu.

C.- Armes rapportées d'Espagne. MMrs Rollin et Feuardent, fer. Geslin, Juillet 1870. (Fig. 3).

a- L.: 39; L. pointe: 9 .

b. L.: 48 ; L. pointe: 6 .

Lieu de conservation actuel inconnu.

\footnotetext{
6 Pour leur rôle dans la vente d'objets chypriotes, voir Olivier MASSON, «La dispersion des antiquités chypriotes: les deux collections Cesnola», CEC, Cahier 25, 1996, I, p. 3-27 (en part. p. 4) et Annie CAUBET et Marielle PIC, «Un culte hathorique à Kition-Bamboula», Archéologie au Levant, Recueil R. Saidah, Lyon, 1982, p. 247 ; et sur les objets égyptiens, voir, par exemple Soheir BAKHOUM et Marie-Christine HELLMANN, «Wilhelm Froehner, le commerce et les collections d'antiquités égyptiennes», Journal des Savants, 1992, p. 159 et 167, n. 69. Sur Felix Feuardent qui, associé avec son gendre Rollin, avait la «grande maison de vente» ... «où sont passés tous les grands archéologues du siècle et du monde»: Gustave SCHLUMBERGER, Mes souvenirs, Paris, 1934, I, p. 177-178.
} 


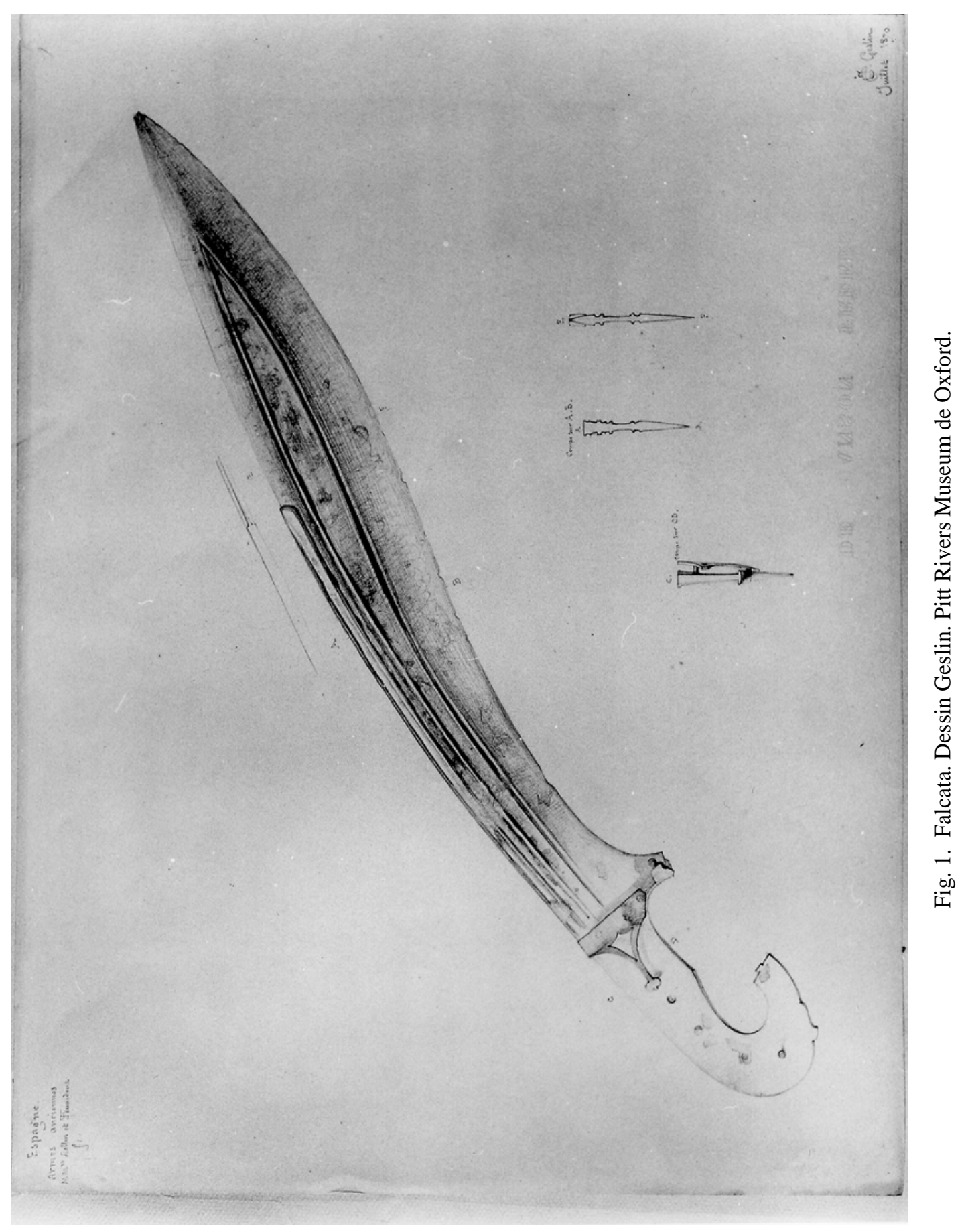




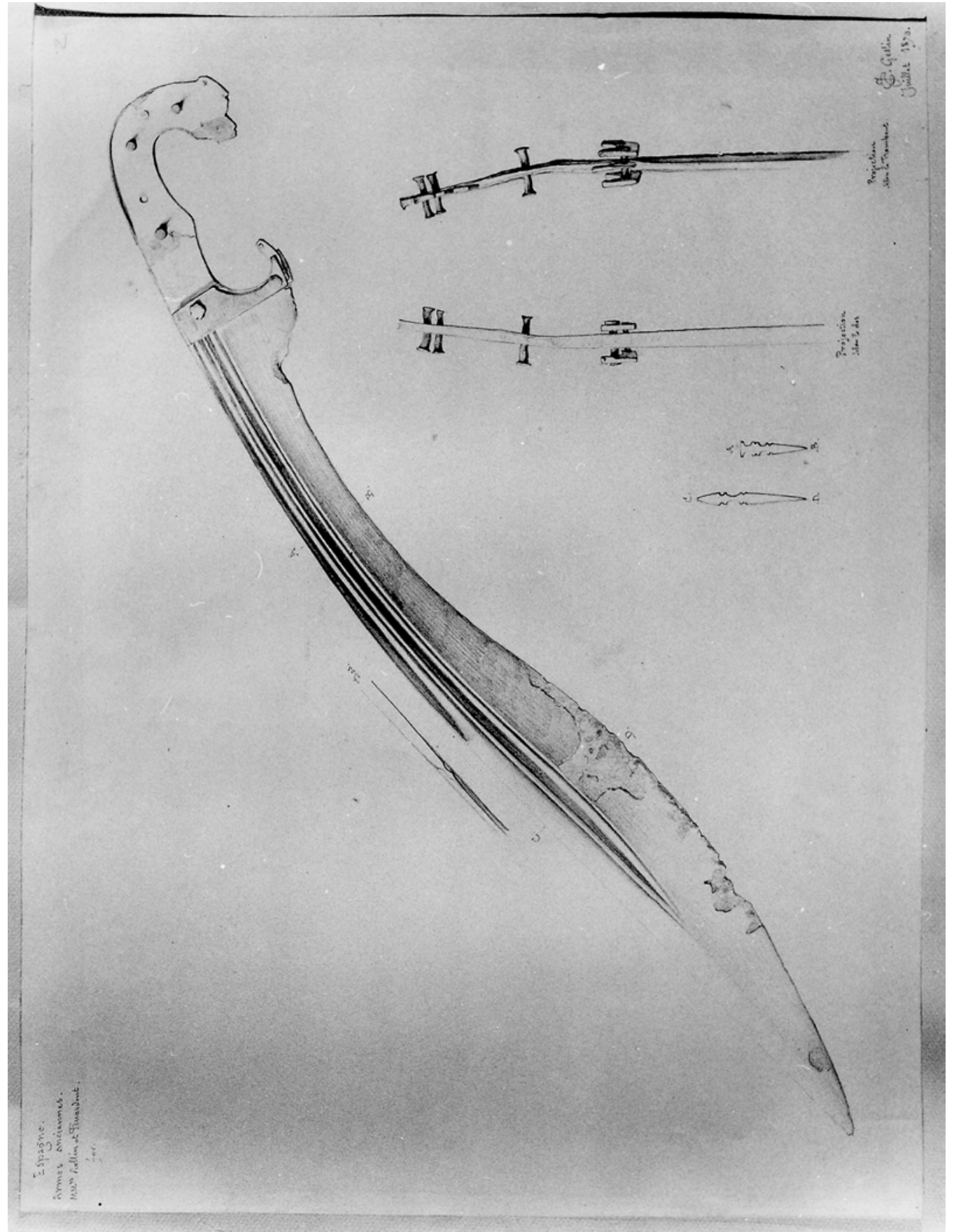

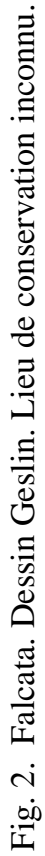




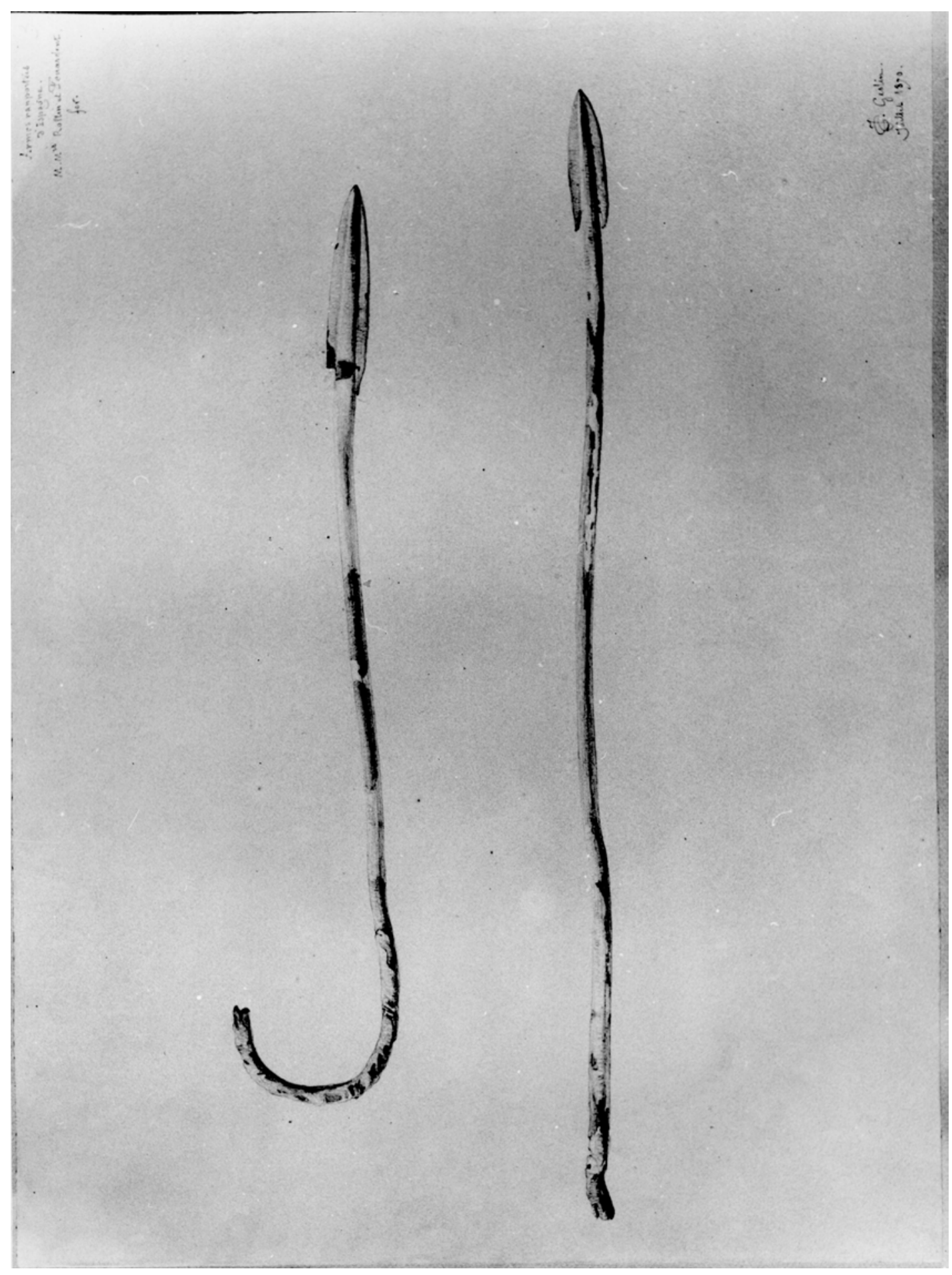

Fig. 3. Fragments de soliferrea. Dessin Geslin. Lieu de conservation inconnu. 
D.- Espagne, armes anciennes, MMrs Rollin et Feuardent, fer. Geslin, Juillet 1870. (Fig. 4).

L.: 23, 8; 46, 6; 38, 3; 27, 7. 1.: A/B: 2, 8; C/D: 3, 3; E/F: 3, 2; G/H: 3, 2.

La première pièce à gauche est conservée aujourd'hui au Pitt Rivers Museum, inv. 1884.120.35. Le lieu de conservation des autres pièces est inconnu.

E.- Espagne, armes anciennes, MMrs Rollin et Feuardent, fer. Geslin, Juillet 1870. (Fig. 5).

a- L.: 34, 5; larg. max: 6, 5 .

b- L.: 33,2 ; larg. max.: 5,7 .

Aujourd'hui au Pitt Rivers Museum, inv. 1884.121.23 et 1884.121.22.

Ainsi toutes les pièces dessinées avec talent et rigueur par Geslin n'ont pas été retrouvées. L'ensemble conservé aujourd'hui au Pitt Rivers Museum d'Oxford contient seulement une des falcatas (Fig. 1), deux poignards (Fig. 5) et une pointe de lance courte (Fig. 4a); manquent une autre falcata (celle de la Fig. 2), les deux fragments de soliferrea (Fig. 3a et b) et les trois lances de grande taille mais en mauvais état de conservation (Fig. 4b, c et d). La sélection a une certaine cohérence dans la mesure où ont été retenues les pièces les mieux conservées et que manquent les trois lances les plus abimées et les fragments de soliferrea, ce qui témoigne d'un esprit de collectionneur particulièrement avisé lors de l'acquisition d'une partie du lot (Fig. 6).

Le Musée Pitt Rivers d'Oxford ne possède pas d'importantes archives sur ces pièces ${ }^{7}$, et nous savons seulement qu'elles faisaient partie de la collection que le général et archéologue Pitt Rivers constitua et donna finalement à l'Université d'Oxford en 1884. ${ }^{8}$ Cette collection comptait 20.000 objets, acquis auprès de nombreux collectionneurs, de plusieurs antiquaires et lors de ventes publiques. Avant la création du Musée, l'ensemble avait été envoyé par Pitt Rivers aux Bethnal Green/South Kensington Museums entre 1874 et 1884, année de la donation et du transfert à Oxford ${ }^{9}$.

\section{DES ARMES D’ANDALOUSIE.}

L'ensemble dessiné par Geslin présente une grande cohérence avec le matériel provenant du Sud-Est de la Péninsule Ibérique et d'Andalousie et en particulier il s'intègre parfaitement aux séries d'armes trouvées par Maraver à Almedinilla en $1867^{10}$, comme on peut le voir dans les répertoires, en particulier dans celui de W. Schüle ${ }^{11}$. Toutefois il est nécessaire de

7 Nous remercions pour son amabilité Helen Mowat, qui a autorisé l'un d'entre nous - F. Q.- à étudier ces armes en 1988, Marina de Alarcón, qui nous a permis d'accéder aux archives du Pitt Rivers Museum et Kate White, du service photographique de cette institution.

8 Voir Mark BOWDEN, Pitt Rivers. The life and archaeological work of Lieutenant-General Augustus Henry Lane Fox Pitt Rivers, DCL, FRS, FSA, Cambridge 1991. Ainsi souligne-t-il: «The name Pitt Rivers is now associated with a magnificiently eccentric musuem in Oxford and, for many people, with the memory of an equally strange Museum in the depths of rural Dorset [...] he was a soldier, landowner, archaeologist and government inspector; a splendid Victorian autocrat who determined to carry out scientific research and to that end caused all kinds of excavations and other activities to be undertaken. His burning desire to educate 'the masses' was the driving force behind his endeavour, for Pitt Rivers was a political animal; but it was as an archaeologist thet he made his greatest impact [...] The General has been called 'the father of scientific archaeology' [...]» (Ibidem p. 1).

9 Sur la formation de la collection et du musée qui porte son nom et sur le goût marqué de Pitt Rivers pour les armes, voir BOWDEN, 1991, passim et en particulier pp. 47 ss.

10 Luis MARAVER Y ALFARO, «Descubrimientos arqueológicos en Almedinilla», dans Revista de Bellas Artes $e$ histórico arqueológica, $2^{\mathrm{a}}$ serie, vol. 2, 1867, pp. 9-10 et 307-328.

11 Wilhem SCHÜLE, Die Meseta Kulturen der Iberischen Halbinsel, 1969, pl. 72-81. 


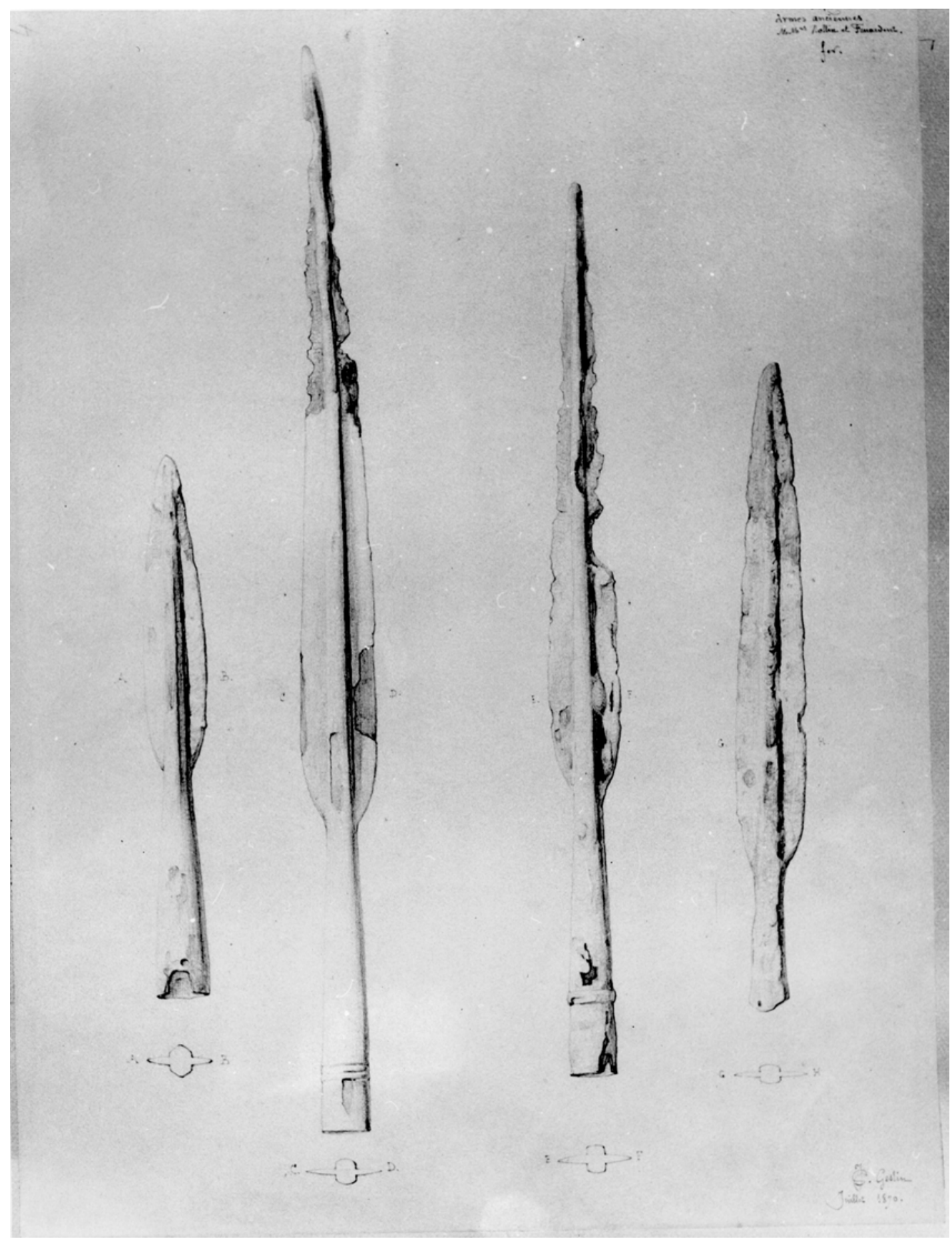

Fig. 4. Pointes de lance. Dessin Geslin. A gauche, Pitt Rivers Museum de Oxford; les trois autres, lieu de conservation inconnu. 

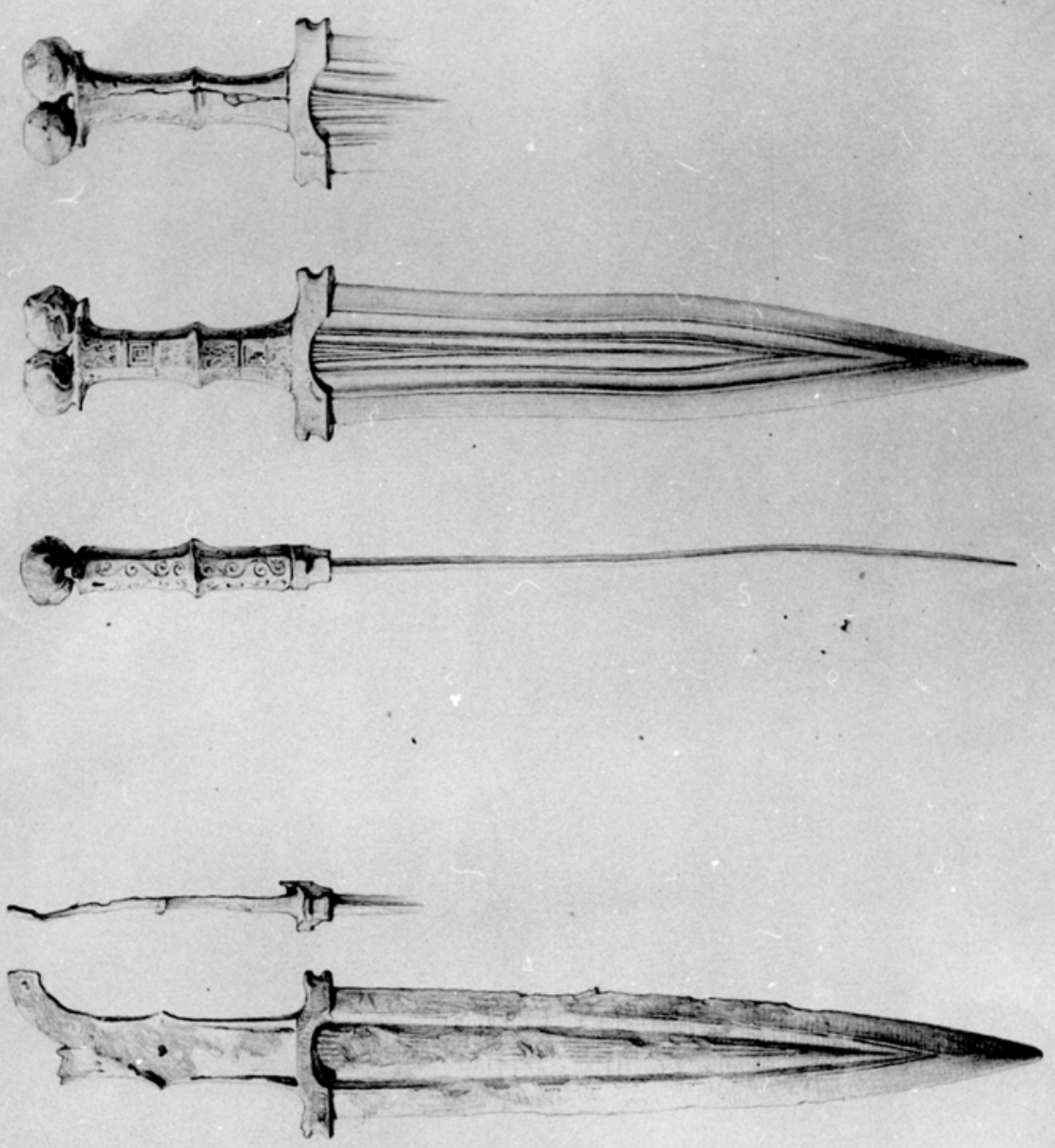

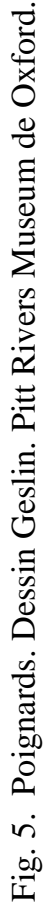

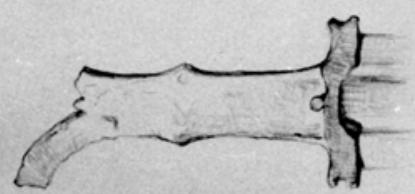




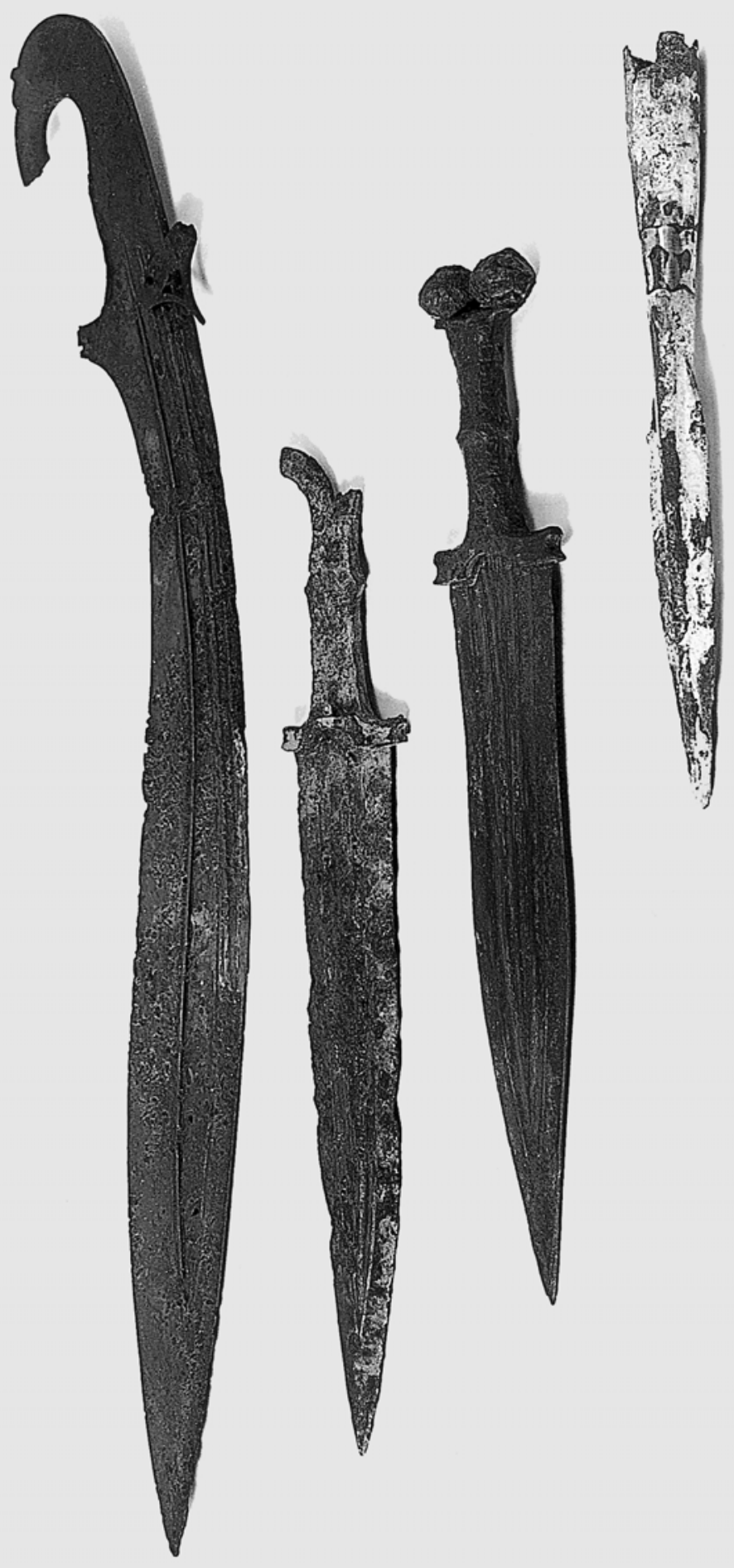

Fig. 6. Le lot d'armes ibériques conservées au Pitt Rivers Museum de Oxford. 
relever que presque toutes les armes ibériques des musées et collections (comme le Museo Arqueológico Nacional de Madrid, le British Museum et le Pitt Rivers Museum) et provenant de fouilles anciennes, indiquent 'Almedinilla' comme lieu de découverte, comme si ceci constituait un sceau d'authenticité et de qualité. Mais si les armes ibériques des Musées et collections européennes provenaient vraiment toutes de Almedinilla, nous aurions une liste plus longue que celle des arme découvertes par Maraver ; cette question récemment soulevée reste ouverte ${ }^{12}$.

La falcata conservée au Pitt Rivers (Fig. 1 y Fig. 6) présente un caractère typique des falcatas andalouses, la garde de l'épée qui se termine en une palmette stylisée d'origine grecque et que les Ibères ont copiée pour orner des épées à pommeau en (forme de) fronton et quelques falcatas ${ }^{13}$. La taille et les proportions de la lame coïncident avec celles de la plupart des épées de la région de Cordoue. La seconde falcata (Fig. 2), dont nous ignorons aujourd'hui la localisation, appartient au même ensemble culturel, de même que les fragments de soliferrea (Fig. 3) et de lances (Fig. 4) et surtout les poignards (Figs. 5 y 6). En effet, le premier est un poignard à pommeau en (forme de) fronton avec languettes divergentes du type Quesada $\mathrm{IB}^{14}$ caractéristique de l'Andalousie et du Sud-Est et que l'on retrouve à l'identique à Almedinilla. ${ }^{15}$ Le second est un poignard à poignée taillée en facettes ${ }^{16}$ du type IIA4; ce type est très rare, mais ses traits se retrouvent attestés à Almedinilla dans une épée courte ${ }^{17}$ et au Cerro de la Mora dans la province voisine de Grenade ${ }^{18}$. Bien que individuellement chacune de ces pièces puisse venir de n'importe quel point du monde ibérique, un tel ensemble d'armes qui compte les deux types de poignards, les soliferrea et les falcatas décrits plus haut ne semble pouvoir avoir été trouvé que dans la Haute Andalousie et la provenance indiquée dans l'inventaire du Pitt Rivers Museum est parfaitement crédible.

La falcata et le poignard à poignée taillée en facettes et antennes atrophiées conservés au Pitt Rivers Museum ont été l'objet, vers 1955, d'une analyse métallographique publiée par H.H. Coghlan: «Etruscan and Spanish swords of iron», Sibrium 3, 1956-57, p. 167-171. Selon ce travail, déjà ancien, la falcata possède une couche extérieure de magnétite bleu-noire, très protectrice contre la corrosion. Le noyau est un fer forgé de qualité relativement basse à faible teneur en carbonne, contenant des impuretés, et il n'y esut pas d'essai pour durcir par carbonisation le fil. Les cannelures ont été réalisées en coupant (o bien en incisant) le métal. Le poignard est lui aussi couvert d'une couche de magnétite, mais le noyau contient beaucoup plus de carbonne que la falcata au point que l'on peut parler d'«acier doux».

FERNANDO QUESADA SANZ

Universidad Autónoma, Madrid

PIERRE ROUILLARD

CNRS, Maison René Ginouvès, Archéologie et Ethnologie, Nanterre

12 Desiderio VAqueRIZO, «Armas de hierro procedentes de la necrópolis ibérica de 'Los Collados' (Almedinilla, Córdoba)», dans Saguntum 22, 1989, p. 227-230 ; Fernando QUESADA, «Notas sobre el armamento ibérico de Almedinilla», Anales de Arqueología Cordobesa, 3, 1992, pp. 113-135.

13 Fernando QUESADA, El armamento ibérico. Estudio tipológico, geográfico, funcional, social y simbólico de las armas en la Cultura Ibérica (siglos VI-I a.C.), Monographies Instrumentum, 3, Montagnac 1997, p. 103 y Fig. 47 ; voir aussi Fernando QUESADA, «Nuevos puñales ibéricos en Andalucía. (I). Puñales de Frontón», Gladius, 19, 1999.

14 QUESADA, El armamento ibérico..., p. 280-281. Le poignard du Pitt Rivers Museum a été publié dans ce même ouvrage fig. 168.

15 Ibidem, p. 857, no. cat. 909, et SCHÜLE, Die Meseta Kulturen... pl. 72.1.

16 QuESADA, El armamento ibérico... p. 280 ss. et p. 285, fig. 168.

17 Ibidem, p. 848, no. cat. 906.

18 Ibidem, p. 208 ss. (sur le type IV) et p. 849, no. cat. 1394. 